\title{
EXPLORING THE PHYSIOLOGICAL MECHANISMS AND ECOLOGICAL CONSEQUENCES OF ENERGETIC TRADEOFFS: AN INTEGRATIVE STUDY OF THE INFLUENCES OF AVIAN MALARIAL INFECTION ON THERMOGENIC PERFORMANCE
}

\author{
MATTHEW CARLING $\uparrow$ UNIVERSITY OF WYOMING $\uparrow$ LARAMIE, WY
}

\section{$\downarrow \quad$ INTRODUCTION}

Survival in variable environments often requires careful allocation of resources to competing physiological and behavioral functions. Because these competing processes often have additive energetic costs (Hawley et al. 2012), a limited resource pool forces individuals to make difficult trade-off decisions regarding energetic investments (Lochmiller and Deerenberg 2000). These trade-offs are a cornerstone of life-history theory that is aimed at determining the optimal allocation strategies in variable environments (Ricklefs and Wikelski 2002), and understanding their physiological and ecological consequences has renewed poignancy in the face of the unprecedented rate of anthropogenic environmental change occurring across the planet.

One such trade-off that is of primary ecological importance is related to immune function. Mounting an immune response is an energetically demanding process that potentially draws resources from other energetically expensive activities, such as thermoregulation in small endotherms (Sheldon and Verhulst 1996, Lochmiller and Deerenberg 2000, Norris and Evans 2000). The consequences of this trade-off between immune function and thermoregulatory ability should be especially acute in high-elevation habitats, because the capacity for aerobic thermogenesis is impaired under hypoxic conditions (Rosenmann and Morrison 1975, Chappell et al. 2007). Thus, even in the absence of an immune challenge, small endotherms living at high altitude are faced with a double bind because their thermogenic capacity is compromised under conditions where thermoregulatory demands are especially severe. Consistent with these expected effects on fitness, survivorship studies of high-altitude endotherms have established strong empirical links between thermogenic capacity and survival in the wild (Hayes and O'Connor 1999). Infection can exacerbate the effects of hypoxia by further compromising thermogenic performance in an environment where any impairment of thermoregulatory capabilities is likely to have profound effects on individual survival, and potentially the persistence of entire populations.

In light of the potential tradeoffs between immune function and thermoregulatory ability, one alarming trend is the upslope movement of pathogens as their vectors track changing global temperatures and other anthropogenic disturbances. One wellknown example of altitudinal movement of an important wildlife disease is avian malaria. Malarial parasites are transmitted by mosquito vectors, and several lines of evidence suggest that global climate change is influencing their distributions, exposing naïve avian populations to parasites with devastating consequences (Freed et al. 2005, Garamszegi 2011, Loiseau et al. 2012). For example, populations of Hawaiian honeycreepers, having evolved for approximately 5 million years in the absence of avian 
malaria (Fleischer et al. 1998), were decimated when the parasite and their vectors were introduced (Dobson and May 1991, McCallum and Dobson 1995). Historically, honeycreeper populations at higher elevations have been spared because they resided in habitats that were too cool to sustain populations of mosquitos. Recently, however, increasing temperatures on the island of Hawaii have resulted in a general upward movement of parasites and vectors, resulting in a doubling in prevalence, over a decade, of avian malaria in birds found at $1900 \mathrm{~m}$ in elevation (Freed et al. 2005). While the general trend is toward warmer temperatures, climate change has also resulted in an increased frequency of severe weather events (e.g., Petoukhov et al. 2013). These extreme weather events may place a premium on thermoregulatory ability, particularly for non-migratory animal species that cannot escape to locations with more benign winter conditions.

Despite the intuitive connections among immunity/thermoregulatory energetic trade-offs, the severity of thermoregulatory challenges in highelevation habitats, and the growing threat of the invasion by novel pathogens, no previous study has systematically explored the extent, mechanisms and consequences of these interactions within an integrated framework. Grand Teton National Park (GTNP) and the surrounding areas provide an ideal natural laboratory to explore these interconnections. Our focal species (see below) occur in relatively high densities across a broad altitudinal gradient, as well as in both disturbed and undisturbed habitats. Taken together, these factors suggest strong spatial patterns in disease prevalence (e.g., Jones et al. in press) and a high likelihood that individual birds will face decisions about where to allocate their limited energetic resources. Here we describe an integrative study that will take advantage of this natural laboratory to examine both physiological underpinnings and ecological consequences of energetic trade-offs between immune function and thermoregulatory performance in passerine birds. We will focus on common species of resident birds, Redbreasted Nuthatch (Sitta canadensis), Mountain Chickadee (Poecile gambeli) and Dark-eyed Junco (Junco hyemalis). These species are terrific study organisms for numerous reasons: 1) they are common (P. gambeli and $S$. canadensis) or abundant ( $J$. hyemalis) in GTNP according to the GTNP Bird Checklist; 2) they are all year-round residents and therefore need to cope with changing weather patterns in each season; and 3) previous work has shown populations of each can be infected with avian hematozoa, which cause avian malaria, at relatively high rates (e.g., $\sim 20 \%$ in $S$. canadensis, $35 \%$ in $P$. gambeli, [Grenier et al. 1975] $\sim 67 \%$ in J. hyemalis [Deviche et al. 2001]).

This work will not only have important implications for our understanding of nature and extent of energetic trade-offs, but it also has important long-term conservation implications. From this perspective, our studies of the spatial distributions of avian malaria parasites will provide a crucial first step toward developing an understanding of how disease prevalence is distributed in the region, providing a baseline against which to measure future patterns of prevalence. Our physiological studies of energetic tradeoffs and the effects of disease state on thermogenic performance will in turn illuminate the potential ecological consequences of spatial variation in malaria infection rates.

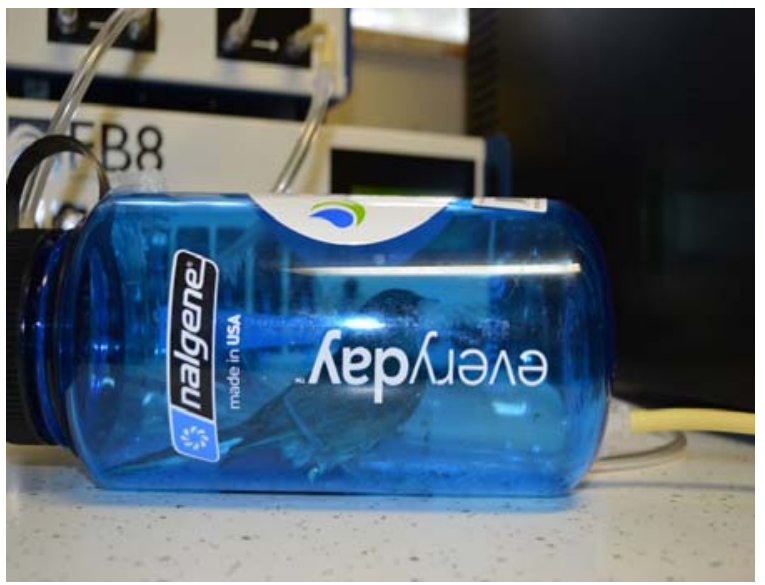

Figure 1. Dark-eyed Junco in metabolic chamber.

The primary goal of our proposal is to investigate the energetic trade-offs between thermoregulation and immune function in several species of Passerine birds. Conducting this research in wild populations allows us the ability to address how birds deal with chronic pathogenic infection, as opposed to LPS injection that primarily simulates an acute response (e.g., Owen-Ashley and Wingfield 2007).

\section{$\uparrow \quad$ METHODS}

We measured a number of metabolic parameters to assess thermogenic performance and baseline energetic costs associated with infection status. Specifically, we used open-flow respirometry to measure: 1) thermogenic capacity [cold-induced summit metabolic rate (SMR) in heliox $(21 \% \mathrm{O} 2,79 \%$ $\mathrm{He})$; 2) resting metabolic rate (RMR); and 3) metabolic scope $[\mathrm{MS}=(\mathrm{SMR}-\mathrm{RMR})]$. These parameters are commonly used to assess thermoregulatory capacities, cold tolerances, and 
baseline energetic costs in birds (Swanson 2010). RMR measurements were made over night using ambient air in a temperature-controlled chamber that was held at temperatures within the thermoneutral zone for small passerines $\left(28^{\circ} \mathrm{C}-\right.$ King and Swanson 2013). We estimated RMR as the minimum O2 consumption averaged over a 10-min period. Measurements of SMR were made the following day using a heliox atmosphere $(21 \% \mathrm{O} 2,79 \% \mathrm{He})$ in temperature-controlled chambers that were maintained from $0^{\circ} \mathrm{C}$ to $-5^{\circ} \mathrm{C}$. Rates of heat loss in heliox are several times greater than in ambient air, which makes it possible to elicit VO2 max without risking cold injury to experimental animals (Rosenmann and Morrison 1974). We estimated SMR as the maximum $\mathrm{O} 2$ averaged over a 5 -min period. Similar protocols have been used to elicit SMR in previous studies (Swanson 2001, Liknes et al. 2002, Swanson 2007, Cheviron et al. 2012, 2013, King and Swanson 2013). To control for the effects of body mass on metabolic parameters, all analyses of RMR and SMR were conducted using an ANOVA framework with body mass as a covariate (Packard and Boardman 1988).

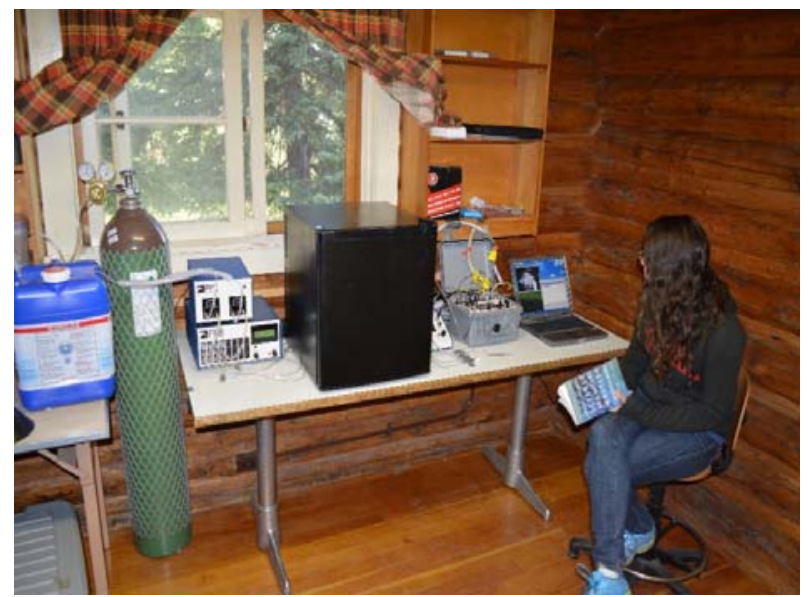

Figure 2. University of Illinois graduate student, Maria Stager, taking metabolic measurements.

DNA was extracted from blood samples using standard protocols. Following DNA extraction, we determined the infection status of each bird using a nested PCR assay (Jones et al. in press). Briefly, we amplified a portion of the cytochrome-b gene of Haemoproteus and Plasmodium, parasites causing avian malaria, and ran the PCR products on an agarose gel. The presence of a band signifies infection, the absence of a band means the bird is not infected with these parasites. In addition to this plus/minus assay, for all positive individuals we will sequence the amplified portion of the cyt-b gene (Jones et al. in press) to identify the lineage of parasite present in each infected bird. The PCR and sequencing assay provide detailed information on the identity of parasites causing avian malaria. It is important to note that at this time we are only proposing to survey infection status, we are not making any assessments of outward disease symptoms. That said, future work may specifically investigate disease manifestation. Similarly, although we have initially focused on avian malaria, it is likely that some birds may be infected with other parasites (i.e., those that do not cause avian malaria) as well.

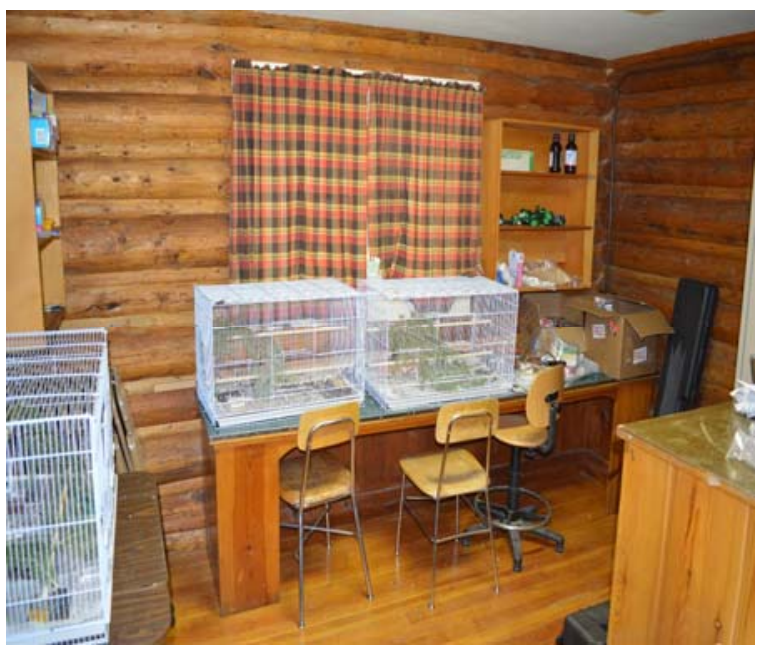

Figure 3. Aviary setup in Berol Lodge.

\section{$\uparrow \quad$ RESULTS}

During the summer of 2013, we measured metabolic parameters (basal metabolic rate and coldinduced summit metabolic rate) in 26 Dark-eyed Juncos, 4 Mountain Chickadees, and 6 Black-capped Chickadees. A majority of these birds have also been screened for the presence of blood-borne malaria parasites (Haemaproteus and Plasomodium), using protocols developed in the Carling Lab. A total of 6 birds tested positive for one or more malaria strains, all of which were juncos. Interestingly, although we sampled juncos from several localities, all of the detected infections occurred at a single site, just outside the boundaries of Grand Teton National Park (Bridger-Teton Nation Forest, Pacific Creek Rd. 43 $53^{\prime} 17.23$ ” N, 11028 ' 29.92” W). At this locality, 40\% of the sampled birds were infected with malaria, suggesting that it may represent a local hotspot for avian malaria infection. Analysis of the influence of malaria infection on metabolic parameters is ongoing, but early analysis suggests that infection status may only have subtle effects on metabolic performance in juncos. 


\section{$\downarrow \quad$ LITERATURE CITED}

Chappell, M. A., K. A. Hammond, R. Cardullo, G. Russell, E. Rezende, and C. Miller. 2007. Deer mouse aerobic performance across altitudes: Effects of developmental history and temperature acclimation. Physiological and Biochemical Zoology 80(6): 652-662.

Cheviron, Z. A., G. C. Bachman, A. D. Connaty, G. B. McClelland, and J. F. Storz. 2012. Regulatory changes contribute to the adaptive enhancement of thermogenic capacity in high-altitude deer mice. Proceedings of the National Academy of Sciences 109: 8635-8640

Cheviron, Z. A., G. C. Bachman, and J. F. Storz. 2013. Contributions of phenotypic plasticity to differences in thermogenic performance between highland and lowland deer mice. Journal of Experimental Biology 216: 11601166.

Deviche, P., E. C. Greiner, and X. Manteca. 2001. Seasonal and age-related changes in blood parasite prevalence in dark-eyed juncos (Junco hyemalis, Aves, Passeriformes). Journal of Experimental Zoology 289: 456466.

Dobson, A. P., and R. M. May. 1991. Parasites, cuckoos, and avian population dynamics. Pages 391-412 In: C. M. Perrins, J. D. Lebreton, and G. J. M. Hirons (eds.), Bird Population Studies: Relevance to Conservation Management, Oxford University Press, Oxford, UK.

Fleischer, R. C., C. E. McIntosh, and C. L. Tarr. 1998. Evolution on a volcanic conveyor belt: Using phylogeographic reconstructions and K-Arbased ages of the Hawaiian Islands to estimate molecular evolutionary rates. Molecular Ecology 7: 533-545.

Freed, L. A., R. L. Cann, M. L. Goff, W. A. Kuntz, and G. R. Bodner. 2005. Increase in avian malaria at upper elevation in Hawai'i. Condor 107: 753-764.

Garamszegi, L. Z. 2011. Climate change increases the risk of malaria in birds. Global Change Biology 17: 1751-1759.

Greiner, E. C., G. F. Bennett, E. M. White, and R. F. Coombs. 1975. Distribution of the avian hematozoa of North America. Canadian Journal of Zoology 53: 1762-1787.

Hawley, D. M.., S. E DuRant, A. F. Wilson, J. S. Adelman, and W. A. Hopkins. 2012. Additive metabolic costs of thermoregulation and pathogen infection. Functional Ecology 26: 701-710.
Hayes, J. P., and C. S. O'Connor. 1999. Natural selection on thermogenic capacity of highaltitude deer mice. Evolution 53: 1280-1287.

Jones, M. R., Z. A. Cheviron, and M. D. Carling. 2013. Spatial patterns of avian malaria prevalence in Zonotrichia capensis on the western slope of the Peruvian Andes. The Journal of Parasitology 99: 903-905.

King, M. O., and D. L. Swanson. 2013. Activation of the immune system incurs energetic costs but has no effect on the thermogenic performance of house sparrows during acute cold challenge. Journal of Experimental Biology 216: 2097-2102.

Liknes, E. T., S. M. Scott, and D. L. Swanson. 2002. Seasonal acclimatization in the American Goldfinch revisited: To what extent do metabolic rates vary seasonally? Condor 104: 548-557.

Lochmiller, R. L., and C. Deerenberg. 2000. Tradeoffs in evolutionary immunology: Just what is the cost of immunity? Oikos 88: 87-98.

Loiseau, C., R. J. Herrigan, A. J. Cornel, S. L. Guers, M. Dodge, and T. Marzec, et al. 2012. First evidence and predictions of Plasmodium transmission in Alaskan bird populations. PLoS One 7: e44729.

McCallum, H., and A. Dobson. 1995. Detecting disease and parasite threats to endangered species and ecosystems. Trends in Ecology \& Evolution 10: 190-194.

Owen-Ashley, N. T., and J. C. Wingfield. 2007. Acute phase responses of passerine birds: Characterization and seasonal variation. Journal of Ornithology 148: 583-591.

Packard, G. C., and T. J. Boardman. 1988. The misuse of ratios, indices, and percentages in ecophysiological research. Physiological Zoology 61: 1-9.

Petoukhov, V., S. Rahmstorf, S. Petri, and H. J. Schellnhuber. 2013. Quasiresonant amplification of planetary waves and recent Northern Hemisphere weather extremes. Proceedings of the National Academy of Sciences 110: 5336-5341.

Ricklefs, R. E., and M. Wikelski. 2002. The physiology/life-history nexus. Trends in Ecology and Evolution. 17: 462-468.

Rosenmann, M., and P. Morrison. 1974. Maximum oxygen consumption and heat loss facilitation in small homeotherms by He-O*. Am. Journal of Physiology 226:490-495.

Sheldon, B. C., and S. Verhulst. 1996. Ecological immunology: Costly parasite defences and trade-offs in evolutionary ecology. Trends in Ecology and Evolution 11: 317-321. 
Swanson, D. L. 2001. Are summit metabolism and thermogenic endurance correlated in winteracclimatized passerine birds? Journal of Comparative Physiology B 171: 475-481.

Swanson, D. L. 2007. Cold hardiness and summit metabolism in North American kinglets during fall migration. Acta Zoologica Sinica 53: 600-606.

Swanson, D. L. 2010. Seasonal metabolic variation in birds: Functional and mechanistic correlates. Current Ornithology 17: 75-129. 